Proceedings of XIX International Scientific Conference "New Technologies and Achievements in Metallurgy, Material Engineering, Production Engineering and Physics", Częstochowa, Poland, June 7-8, 2018

\title{
Non-Adiabatic Effects in Superconducting Intermetallic Borocarbides
}

\author{
A.P. Durajski ${ }^{a, *}$, K.M. SkOczylas ${ }^{b}$ And A.E. AuguściK ${ }^{a}$ \\ ${ }^{a}$ Institute of Physics, Częstochowa University of Technology, al. Armii Krajowej 19, 42-200 Częstochowa, Poland \\ ${ }^{b}$ Institute of Physics, Jan Długosz University in Częstochowa, al. Armii Krajowej 13/15, 42-200 Częstochowa, Poland
}

\begin{abstract}
In this work, we systematically study the non-adiabatic effects on the superconductivity of selected intermetallic borocarbides. Using the strong-coupling Eliashberg theory with and without lowest-order vertex correction we find that for a fixed experimental value of critical temperature the Coulomb pseudopotential can take different values, however, the superconducting order parameter remains practically unaffected. From the physical point of view, it means that in the investigated superconductors the lowest-order vertex correction does not play a significant role.
\end{abstract}

DOI: 10.12693 /APhysPolA.135.276

PACS/topics: superconductivity, borocarbides, non-adiabatic effects, vertex corrections

\section{Introduction}

The intermetallic borocarbides with formula $\mathrm{RT}_{2} \mathrm{~B}_{2} \mathrm{C}$ (in which $\mathrm{R}$ is a rare earth element and $\mathrm{T}$ denotes a transition metal) are subject of extensive theoretical as well as experimental attention due to great variety of their fascinating physical properties. Especially, in the certain combination of $\mathrm{R}$ and $\mathrm{T}$ elements, the magnetic and superconducting ordering can coexist $[1,2]$. In particular, the magnetic moments come from the rare earths and the transition metals specify the density of conduction electrons [3]. The conventional phonon-mediated superconductivity with a strong electron-phonon coupling strength in these materials is evidenced by specific heat and boron isotope effect measurements [4-6]. These compounds show a layered structure, and therefore they are considered as possibly close to cuprates [7]. The evidence for a multi-band nature of the superconductivity in $\mathrm{YNi}_{2} \mathrm{~B}_{2} \mathrm{C}$ and $\mathrm{LuNi}_{2} \mathrm{~B}_{2} \mathrm{C}$ was reported among others in the papers $[8,9]$. One of the main features of these materials is the presence of two energy gaps which, however, vanish at the same critical temperature [10]. Our previous theoretical work on intermetallic borocarbides $\mathrm{YPd}_{2} \mathrm{~B}_{2} \mathrm{C}, \mathrm{YPt}_{2} \mathrm{~B}_{2} \mathrm{C}$ and $\mathrm{LaPt}_{2} \mathrm{~B}_{2} \mathrm{C}$ [11] shows that the experimental values of the critical temperature cannot be properly reproduced using the commonly accepted value of the Coulomb pseudopotential. Moreover, we proved that the values of universal ratios of conventional superconductivity appearing in the BardeenCooper-Schrieffer (BCS) theory of superconductivity [12] are inconsistent with our results obtained in the framework of the Eliashberg formalism [14]. The observed differences are connected with the strong/medium-coupling and retardation effects existing in the investigated mate-

*corresponding author; e-mail: adurajski@wip.pcz.pl rials. Thus, in the light of our previous work, herein we have studied the influence of the non-adiabatic effects on the superconducting properties of $\mathrm{YPd}_{2} \mathrm{~B}_{2} \mathrm{C}, \mathrm{YPt}_{2} \mathrm{~B}_{2} \mathrm{C}$ and $\mathrm{LaPt}_{2} \mathrm{~B}_{2} \mathrm{C}$. In particular, we take into account the behavior of the Coulomb pseudopotential and superconducting order parameter.

This paper is organized as follows. In Sect. 2, we introduce the theoretical model used to determine the quantities characterizing the superconductors. In Sect. 3, we report and compare the investigated thermodynamic parameters of superconducting $\mathrm{YPd}_{2} \mathrm{~B}_{2} \mathrm{C}, \mathrm{YPt}_{2} \mathrm{~B}_{2} \mathrm{C}$ and $\mathrm{LaPt}_{2} \mathrm{~B}_{2} \mathrm{C}$. In particular, we discuss the validity of the conventional Migdal-Eliashberg theory and we examine its effect of lowest-order vertex correction on the Coulomb pseudopotential and superconducting order parameter. Finally, we give a summary of this study in Sect. 4.

\section{Theoretical model and computational methods}

The thermodynamic properties of phonon-mediated superconductors can be accurately described within the framework of the Migdal-Eliashberg theory which is one of the most powerful and versatile approaches to study of superconductivity $[14,15]$. Due to the absence of evidences for multi-band character of superconducting state in $\mathrm{YPd}_{2} \mathrm{~B}_{2} \mathrm{C}, \mathrm{YPt}_{2} \mathrm{~B}_{2} \mathrm{C}$, and $\mathrm{LaPt}_{2} \mathrm{~B}_{2} \mathrm{C}$ we conducted our investigations in the framework of an isotropic single-band Eliashberg model. For the superconducting order parameter function $\varphi_{n}=\varphi\left(\mathrm{i} \omega_{n}\right)$ and for the electron mass renormalization function $Z_{n}=Z\left(\mathrm{i} \omega_{n}\right)$ in the imaginary-frequencies axis formulation the Eliashberg equations take the following form $[14,16]$ :

$$
\varphi_{n}=\pi T \sum_{m=-M}^{M} \frac{\lambda_{n, m}-\mu^{*} \theta\left(\omega_{c}-\left|\omega_{m}\right|\right)}{\sqrt{\omega_{m}^{2} Z_{m}^{2}+\varphi_{m}^{2}}} \varphi_{m}
$$




$$
Z_{n}=1+\frac{\pi T}{\omega_{n}} \sum_{m=-M}^{M} \frac{\lambda_{n, m}}{\sqrt{\omega_{m}^{2} Z_{m}^{2}+\varphi_{m}^{2}}} \omega_{m} Z_{m}
$$

where the electron-phonon interaction pairing kernel is defined as:

$$
\lambda_{n, m}=2 \int_{0}^{\infty} \mathrm{d} \omega \frac{\omega}{\left(\omega_{n}-\omega_{m}\right)^{2}+\omega^{2}} \alpha^{2} F(\omega) .
$$

Symbol $\mu^{*}$ denotes the Coulomb pseudopotential. The Heaviside function is given by $\theta$ and the cut-off frequency $\left(\omega_{c}\right)$ equals ten times the maximal phonon frequency.

The application of the above Eliashberg equations to describe the electron-phonon superconductivity is justified for systems in which the value of the Debye phonon frequency $\left(\omega_{D}\right)$ to the Fermi energy $\left(\varepsilon_{F}\right)$ ratio is negligible $\omega_{D} / \varepsilon_{F} \approx 0$. Otherwise the Eliashberg equations should be generalized by taking into account the lowest-order vertex correction for electron-phonon interactions which describes the non-adiabatic effects which can appreciably raise $T_{c}$ and lead to a number of new effects for both the superconductive and normal state [17]. In high-temperature cuprates and fullerenes the value of ratio $\omega_{D} / \varepsilon_{F}$ is of the order of 0.1 and therefore it is necessary to include vertex corrections in the self-energy [18-20]. The investigated in this paper compounds have a layered structure, and therefore they are considered as close to cuprates. Moreover, the ratio $\omega_{D} / \varepsilon_{F}$ is of the order of 0.01 and therefore it would seem that the vertex corrections due to the breakdown of Migdal's theorem for the electron-phonon interactions should also have important consequences on the thermodynamic properties of superconducting state. The generalization Eliashberg equations can be written in the following form $[18,19,21-23]$ :

$$
\begin{aligned}
\varphi_{n} & =\pi T \sum_{m=-M}^{M} \frac{\lambda_{n, m}-\mu^{*} \theta\left(\omega_{c}-\left|\omega_{m}\right|\right)}{\sqrt{\omega_{m}^{2} Z_{m}^{2}+\varphi_{m}^{2}}} \varphi_{m}-\frac{\pi^{3} T^{2}}{4 \varepsilon_{F}} \\
& \times \sum_{m=-M}^{M} \sum_{m^{\prime}=-M}^{M} \frac{\lambda_{n, m} \lambda_{n, m^{\prime}}}{\sqrt{\left(\omega_{m}^{2} Z_{m}^{2}+\varphi_{m}^{2}\right)\left(\omega_{m^{\prime}}^{2} Z_{m^{\prime}}^{2}+\varphi_{m^{\prime}}^{2}\right)\left(\omega_{-n+m+m^{\prime}}^{2} Z_{-n+m+m^{\prime}}^{2}+\varphi_{-n+m+m^{\prime}}^{2}\right)}} \\
& \times\left[\varphi_{m} \varphi_{m^{\prime}} \varphi_{-n+m+m^{\prime}}+2 \varphi_{m} \omega_{m^{\prime}} Z_{m^{\prime}} \omega_{-n+m+m^{\prime}} Z_{-n+m+m^{\prime}}-\omega_{m} Z_{m} \omega_{m^{\prime}} Z_{m^{\prime}} \varphi_{-n+m+m^{\prime}}\right],
\end{aligned}
$$

and

$$
\begin{aligned}
Z_{n} & =1+\frac{\pi T}{\omega_{n}} \sum_{m=-M}^{M} \frac{\lambda_{n, m}}{\sqrt{\omega_{m}^{2} Z_{m}^{2}+\varphi_{m}^{2}}} \omega_{m} Z_{m}-\frac{\pi^{3} T^{2}}{4 \varepsilon_{F} \omega_{n}} \\
& \times \sum_{m=-M}^{M} \sum_{m^{\prime}=-M}^{M} \frac{\lambda_{n, m} \lambda_{n, m^{\prime}}}{\sqrt{\left(\omega_{m}^{2} Z_{m}^{2}+\varphi_{m}^{2}\right)\left(\omega_{m^{\prime}}^{2} Z_{m^{\prime}}^{2}+\varphi_{m^{\prime}}^{2}\right)\left(\omega_{-n+m+m^{\prime}}^{2} Z_{-n+m+m^{\prime}}^{2}+\varphi_{-n+m+m^{\prime}}^{2}\right)}} \\
& \times\left[\omega_{m} Z_{m} \omega_{m^{\prime}} Z_{m^{\prime}} \omega_{-n+m+m^{\prime}} Z_{-n+m+m^{\prime}}+2 \omega_{m} Z_{m} \varphi_{m^{\prime}} \varphi_{-n+m+m^{\prime}}-\varphi_{m} \varphi_{m^{\prime}} \omega_{-n+m+m^{\prime}} Z_{-n+m+m^{\prime}}\right] .
\end{aligned}
$$

The Eliashberg spectral functions $\alpha^{2} F(\omega)$ for the investigated intermetallic borocarbides have been previously calculated by Uzunok et al. [24, 25] using the QuantumESPRESSO package [26, 27].

Like many other complex systems [28-33], the Eliashberg equations are too complicated to solve them in an analytical way. Due to the above, in this study the numerical analysis was performed using a self-consistent iteration methods [34], which were implemented successfully in our previous papers [35-38]. The convergence and precision of presented results are controlled by assuming the high number $(M)$ of the Matsubara frequencies $\omega_{n}=(\pi / \beta)(2 n-1)$, where $n=0, \pm 1, \pm 2, \ldots, \pm M$. Both sets of the Eliashberg equations were solved simultaneously.

\section{Results and discussion}

To correctly evaluate the thermodynamic properties of superconducting state within the framework of the Eliashberg equations, the screened Coulomb interaction (the Coulomb pseudopotential $\mu_{c}^{*}$ ) should be calculated using the condition that the superconducting order parameter $\left(\Delta_{n=1}=\varphi_{n=1} / Z_{n=1}\right)$ vanishes at critical temperature obtained directly from the experimental measurements.

The calculated course of the function $\Delta_{n=1}\left(\mu^{*}\right)$ is presented in Fig. 1.

From the following relation $\left[\Delta_{n=1}\left(\mu^{*}\right)\right]_{T=T}=0$, where $T_{c}$ for $\mathrm{YPd}_{2} \mathrm{~B}_{2} \mathrm{C}, \mathrm{YPt}_{2} \mathrm{~B}_{2} \mathrm{C}$, and $\mathrm{LaPt}_{2} \mathrm{~B}_{2} \mathrm{C}$ equals 21,10 , and $10 \mathrm{~K}$, respectively [39-41], we can conclude that in the framework of the Eliashberg equations 


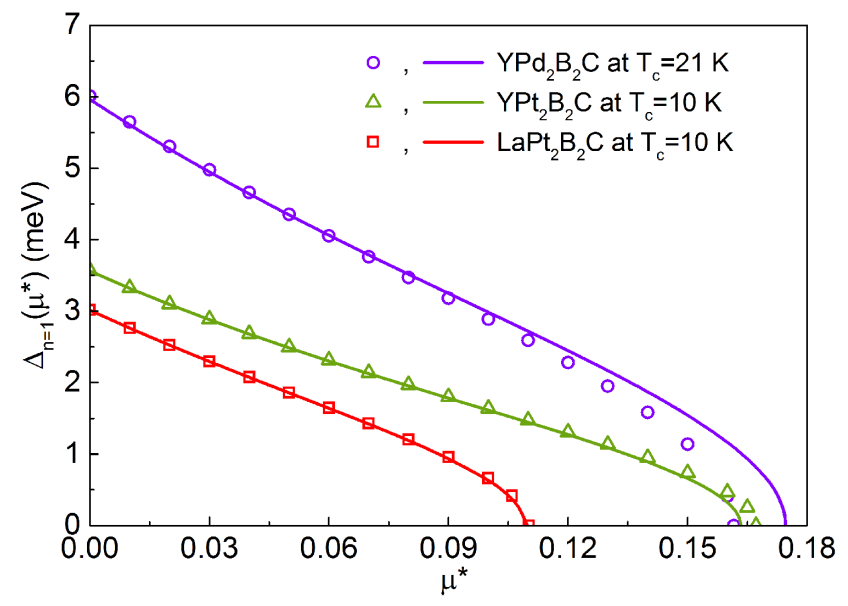

Fig. 1. The dependence of the maximum value of the order parameter on $\mu^{*}$ at $T=T_{c}$. The lines and symbols correspond to the Eliashberg equations with and without lowest-order vertex correction, respectively.

without vertex corrections $\mu_{c}^{*}=0.162$ for $\mathrm{YPd}_{2} \mathrm{~B}_{2} \mathrm{C}$, $\mu_{c}^{*}=0.167$ for $\mathrm{YPt}_{2} \mathrm{~B}_{2} \mathrm{C}$ and $\mu_{c}^{*}=0.110$ for $\mathrm{LaPt}_{2} \mathrm{~B}_{2} \mathrm{C}$. The implementation of the lowest-order vertex correction for electron-phonon interactions into the Eliashberg equations causes a small reduction of $\mu_{c}^{*}$ in the case of $\mathrm{YPt}_{2} \mathrm{~B}_{2} \mathrm{C}$, in the case of $\mathrm{LaPt}_{2} \mathrm{~B}_{2} \mathrm{C}$ we did not see any change and for $\mathrm{YPd}_{2} \mathrm{~B}_{2} \mathrm{C}$ the Coulomb pseudopotential has increased to the value of 0.175 .

In the next step, we examine the influence of the lowest-order vertex correction on the superconducting order parameter. Figure 2 presents the plot of the dependence of the order parameter for the first Matsubara frequency on temperature.

It is clearly visible that the values of order parameter calculated with regard to the vertex corrections are practically identical with the values of $\Delta_{n=1}(T)$ designated within the framework of the classic Eliashberg scheme.

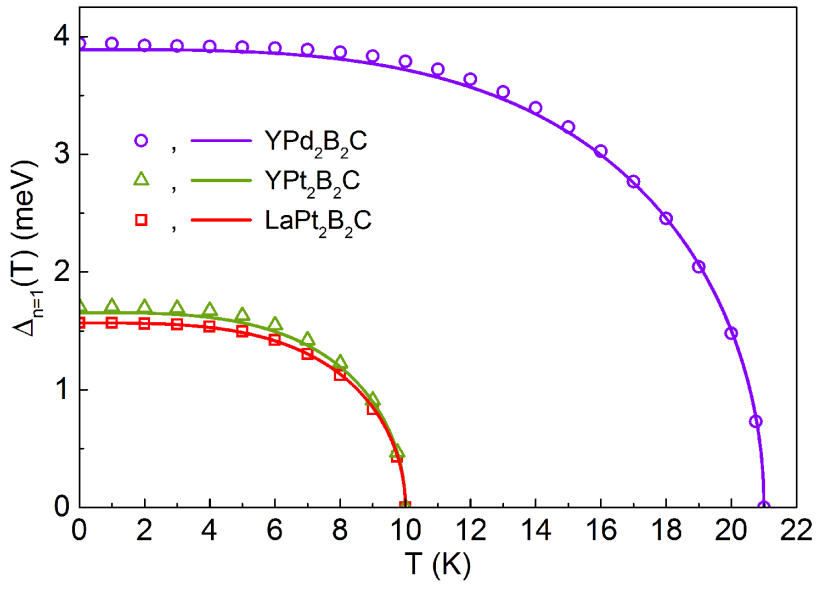

Fig. 2. The temperature dependence of the maximum value of the order parameter. The lines and symbols correspond to the Eliashberg equations with and without lowest-order vertex correction, respectively.
This means that the superconducting properties of intermetallic borocarbides can be successfully obtained in the framework of the conventional Migdal-Eliashberg formalism with the proviso that $\mu_{c}^{*}$ has to be accurately determined. This result is connected with the fact that the electron-phonon vertex correction is shown to be so small, $\omega_{D} / \varepsilon_{F} \approx 0.012$, and can be neglected. Therefore conventional Migdal-Eliashberg formalism is enough to capture the thermodynamics of these materials well.

\section{Conclusions}

In present paper we have carefully studied the nonadiabatic effects in superconducting $\mathrm{YPd}_{2} \mathrm{~B}_{2} \mathrm{C}, \mathrm{YPt}_{2} \mathrm{~B}_{2} \mathrm{C}$ and $\mathrm{LaPt}_{2} \mathrm{~B}_{2} \mathrm{C}$ using the conventional and extended Eliashberg equations. From the numerical calculations described herein, we can conclude that for the fixed experimental values of critical temperature, the lowestorder vertex correction only slightly changes the Coulomb pseudopotential and plays a negligibly small role in the case of description of the superconducting order parameter. On this basis, we proved that the conventional Migdal-Eliashberg formalism is enough to capture the thermodynamics of these materials on the quantitative level.

\section{Acknowledgments}

A.P. Durajski gratefully acknowledges the financial support from the Polish Ministry of Science and Higher Education under the scholarship for young outstanding scientists. All authors are grateful to the Częstochowa University of Technology - MSK CzestMAN for granting access to the computing infrastructure built in the projects No. POIG.02.03.00-00-028/08 "PLATON Science Services Platform" and No. POIG.02.03.00-00$110 / 13$ "Deploying high-availability, critical services in Metropolitan Area Networks (MAN-HA)".

\section{References}

[1] T. Paiva, M.E. Massalami, R.R. dos Santos, J. Phys. Condens. Matter 15, 7917 (2003).

[2] D.H. Galvan, A. Durán, A.P. Amarillas, R. Escudero, Phys. Rev. B 74, 245121 (2006).

[3] K.-H. Müller, V.N. Narozhnyi, Rep. Prog. Phys. 64, 943 (2001).

[4] S.A. Carter, B. Batlogg, R.J. Cava, J.J. Krajewski, W.F. Peck, H. Takagi, Phys. Rev. B 50, 4216 (1994).

[5] J.S. Kim, W.W. Kim, G.R. Stewart, Phys. Rev. B 50, 3485 (1994).

[6] K. Cheon, I. Fisher, P. Canfield, Physica C 312, 35 (1999).

[7] I. Askerzade, Unconventional Superconductors: Anisotropy and Multiband Effects, Springer, Berlin 2012.

[8] S.V. Shulga, S.-L. Drechsler, G. Fuchs, K.-H. Müller, K. Winzer, M. Heinecke, K. Krug, Phys. Rev. Lett. 80, 1730 (1998). 
[9] X. Lu, W.K. Park, S. Yeo, K.-H. Oh, S.-I. Lee, S.L. Bud'ko, P.C. Canfield, L.H. Greene, Phys. Rev. B 83, 104519 (2011).

[10] K.V. Grigorishin, Phys. Lett. A 380, 1781 (2016).

[11] A. Durajski, M. Paliwoda, R. Szczęśniak, Solid State Sci. 61, 215 (2016).

[12] J. Bardeen, L.N. Cooper, J.R. Schrieffer, Phys. Rev. 106, 162 (1957).

[13] J. Bardeen, L.N. Cooper, J.R. Schrieffer, Phys. Rev. 108, 1175 (1957).

[14] G.M. Eliashberg, J. Exp. Theor. Phys. 11, 696 (1960).

[15] J.P. Carbotte, Rev. Mod. Phys. 62, 1027 (1990).

[16] F. Marsiglio, M. Schossmann, J.P. Carbotte, Phys. Rev. B 37, 4965 (1988).

[17] E. Cappelluti, L. Pietronero, Europhys. Lett. 36, 619 (1996).

[18] L. Pietronero, S. Strässler, C. Grimaldi, Phys. Rev. B 52, 10516 (1995).

[19] C. Grimaldi, L. Pietronero, S. Strässler, Phys. Rev. B 52, 10530 (1995).

[20] G.A. Ummarino, in: Emergent Phenomena in Correlated Matter, Vol. 3, Eds. E. Pavarini, E. Koch, U. Schollwöck, Forschungszentrum Jülich GmbH, 2013, Ch. 13.

[21] P. Miller, J.K. Freericks, E.J. Nicol, Phys. Rev. B 58, 14498 (1998).

[22] C. Grimaldi, L. Pietronero, M. Scattoni, Eur. Phys. J. B 10, 247 (1999).

[23] L. Pietronero, S. Strässler, Europhys. Lett. 18, 627 (1992).

[24] H.Y. Uzunok, H.M. Tütüncü, S. Özer, S. Uğur, G.P. Srivastava, Solid State Commun. 206, 1 (2015).

[25] H.M. Tütüncü, H.Y. Uzunok, E. Karaca, G.P. Srivastava, S. Özer, S. Uğur, Phys. Rev. B 92, 054510 (2015).

[26] P. Giannozzi, O. Andreussi, T. Brumme, O. Bunau, M.B. Nardelli, M. Calandra, R. Car, C. Cavazzoni, D. Ceresoli, M. Cococcioni, N. Colonna, I. Carnimeo, A.D. Corso, S. de Gironcoli, P. Delugas, R.A. DiStasio Jr., A. Ferretti, A. Floris, G. Fratesi, G. Fugallo, R. Gebauer, U. Gerstmann, F. Giustino, T. Gorni, J. Jia, M. Kawamura, H.-Y. Ko, A. Kokalj, E. Kkbenli, M. Lazzeri, M. Marsili, N. Marzari, F. Mauri, N.L. Nguyen, H.-V. Nguyen, A.O. de-la Roza, L. Paulatto, S. Ponc, D. Rocca, R. Sabatini, B. Santra, M. Schlipf, A.P. Seitsonen, A. Smogunov, I. Timrov, T. Thonhauser, P. Umari, N. Vast, X. Wu, S. Baroni, J. Phys. Condens. Matter 29, 465901 (2017).
[27] P. Giannozzi, S. Baroni, N. Bonini, M. Calandra R. Car, C. Cavazzoni, D. Ceresoli, G.L. Chiarotti, M. Cococcioni, I. Dabo, A.D. Corso, S. de Gironcoli, S. Fabris, G. Fratesi, R. Gebauer, U. Gerstmann, C. Gougoussis, A. Kokalj, M. Lazzeri, L. Martin-Samos, N. Marzari, F. Mauri, R. Mazzarello, S. Paolini, A. Pasquarello, L. Paulatto, C. Sbraccia, S. Scandolo, G. Sclauzero, A.P. Seitsonen, A. Smogunov, P. Umari, R.M. Wentzcovitch, J. Phys. Condens. Matter 21, 395502 (2009).

[28] M. Ciesielski, T. Blaszczyk, J. Theor. Appl. Mech. 53, 959 (2015)

[29] D. Baleanu, T. Blaszczyk, J. Asad, M. Alipour, Acta Phys. Pol. A 130, 688 (2016).

[30] T. Blaszczyk, M. Ciesielski, Adv. Appl. Math. Mech. 9, 173 (2017).

[31] M.W. Jarosik, R. Szczęśniak, A.P. Durajski, J.K. Kalaga, W. Leonski, Chaos 28, 013126 (2018).

[32] W. Leonski, J.K. Kalaga, R. Szczęśniak, M.W. Jarosik, in: Chaos Theory, Ed. K.A.M. Al Naimee, InTech, Rijeka 2018, p. 119.

[33] R. Gonczarek, M. Krzyzosiak, A. Gonczarek, L. Jacak, Int. J. Mod. Phys. B 29, 1550117 (2015).

[34] R. Szczęśniak, Acta Phys. Pol. A 109, 179 (2006).

[35] R. Szczęśniak, M.W. Jarosik, Solid State Commun. 149, 2053 (2009).

[36] R. Szczęśniak, D. Szczęśniak, E.A. Drzazga, Solid State Commun. 152, 2023 (2012).

[37] M.W. Jarosik, R. Szczęśniak, I.A. Wrona, M. Kostrzewa, Solid State Commun. 250, 5 (2017).

[38] R. Szczeęśniak, E.A. Drzazga, D. Szczęśniak, Eur. Phys. J. B 88, 52 (2015).

[39] H. Bitterlich, W. Loser, H.-G. Lindenkreuz, L. Schultz, J. Alloys Comp. 325, 285 (2001).

[40] F. Yang, N. Tang, J. Wang, W. Qin, Z.-X. Li, J. Luo, J. Phys. Condens. Matter 7, 2369 (1995).

[41] R.J. Cava, B. Batlogg, T. Siegrist, J.J. Krajewski, W.F. Peck, S. Carter, R.J. Felder, H. Takagi, R.B. van Dover, Phys. Rev. B 49, 12384 (1994). 